\title{
Electrocorticography and the early maturation of high-frequency suppression within the default mode network
}

\author{
Kurt E. Weaver, PhD, $1,8,9,11$ Andrew Poliakov, PhD, ${ }^{5}$ Edward J. Novotny, MD, $, 8,8,10$ \\ Jared D. Olson, MD, ${ }^{4,11}$ Thomas J. Grabowski, MD, ${ }^{1,3,8,9,11}$ and Jeffrey G. Ojemann, MD $2,7-11$ \\ Departments of ${ }^{1}$ Radiology, ${ }^{2}$ Neurological Surgery, ${ }^{3}$ Neurology, and ${ }^{4}$ Rehabilitation Medicine, University of Washington, Seattle; \\ Departments of ${ }^{5}$ Radiology, ${ }^{6}$ Neurology, and ${ }^{7}$ Neurosurgery, Seattle Children's Hospital, Seattle; ${ }^{8}$ nntegrated Brain Imaging \\ Center, University of Washington, Seattle; ${ }^{9}$ Graduate Program in Neuroscience, University of Washington, Seattle; ${ }^{10}$ Center for \\ Integrative Brain Research, Seattle Children's Research Institute, Seattle; and ${ }^{11}$ Center for Sensorimotor Neural Engineering, \\ University of Washington, Seattle, Washington
}

OBJECTIVE The acquisition and refinement of cognitive and behavioral skills during development is associated with the maturation of various brain oscillatory activities. Most developmental investigations have identified distinct patterns of low-frequency electrophysiological activity that are characteristic of various behavioral milestones. In this investigation, the authors focused on the cross-sectional developmental properties of high-frequency spectral power from the brain's default mode network (DMN) during goal-directed behavior.

METHODS The authors contrasted regionally specific, time-evolving high gamma power (HGP) in the lateral DMN cortex between 3 young children (age range 3-6 years) and 3 adults by use of electrocorticography (ECoG) recordings over the left perisylvian cortex during a picture-naming task.

RESULTS Across all participants, a nearly identical and consistent response suppression of HGP, which is a functional signature of the DMN, was observed during task performance recordings acquired from ECoG electrodes placed over the lateral DMN cortex. This finding provides evidence of relatively early maturation of the DMN. Furthermore, only HGP relative to evoked alpha and beta band power showed this level of consistency across all participants.

CONCLUSIONS Regionally specific, task-evoked suppression of the high-frequency components of the cortical power spectrum is established early in brain development, and this response may reflect the early maturation of specific cognitive and/or computational mechanisms.

https://thejns.org/doi/abs/10.3171/2017.7.PEDS17269

KEY WORDS electrocorticography; high gamma power; suppression; default mode network; brain development

$\mathrm{M}$ ATURATION of various brain oscillations is a physiological milestone commonly associated with developmental gains in cognitive and behavioral function. ${ }^{16,26}$ One domain of developmental human neurophysiology that remains poorly explored is the maturation of the high-frequency component (i.e., high gamma [HG], 70-200 Hz) of the broadband cortical spectrum. Contemporary explorations in the mature brain have focused on the relationship between evoked high-frequency activ- ity and behavior. This is due in part to observations that task-evoked HG power (HGP) is the best marker of focal cortical activity, ${ }^{18}$ thereby serving as a metric of local computation across a number of functional domains, such as sensory processing, attention, memory, and motor control..$^{15}$ Although the mechanistic and physiological origins of HG signals are debated, ${ }^{5,17}$ it is widely held that high-frequency responses represent some fundamental physiological process of human cortical function. ${ }^{21,28}$

ABBREVIATIONS DMN = default mode network; ECOG = electrocorticography; ERTS = event-related time series; FC = functional connectivity; fMRI = functional MRI; FSL = FMRIB Software Library; HG = high gamma; HGP = HG power; HMC = Harborview Medical Center; ITI = intertrial interval; MNI = Montreal Neurological Institute; rsfMRI = resting-state fMRl; $\mathrm{SCH}=$ Seattle Children's Hospital.

SUBMITTED May 22, 2017. ACCEPTED July 24, 2017.

INCLUDE WHEN CITING Published online December 1, 2017; DOI: 10.3171/2017.7.PEDS17269. 
Subdural electrocorticography (ECoG) is exquisitely sensitive to task-evoked modulation of HGP. By sampling directly from the cortical surface, ECoG is significantly less confounded by cranial muscle artifacts within the same band-limited range and signal degradation that occurs as a function of increasing distance from the electrical source. ${ }^{22}$ ECoG is thus ideally suited to characterize responsive cortical fields during early developmental periods when movement artifacts and task-based noncompliance are significant concerns.

A few ECoG reports on humans have revealed patterns of evoked HGP in very young children that mimic the typical response profiles in adult counterparts. ${ }^{4,14}$ This finding suggests that the physiological mechanisms that generate gains in evoked HGP are likely established early in human brain development.

In contrast, there have been no reported ECoG studies of HGP suppression that is characteristic to the default mode network (DMN) in children. The DMN is a largescale, neocortical network composed of a set of spatially disparate association areas primarily located deeply along the cortical midline and laterally on the parietal surface. ${ }^{3}$ Since the DMN was first conceptualized, ${ }^{25}$ imaging and electrophysiological studies have converged on a number of key characteristics that fundamentally impact cognition and behavior. One feature that has emerged is the observation that the DMN can influence cognitive function through reduced suppression, which is commonly referred to as task-induced deactivation. ${ }^{1}$ Specifically, imaging studies have shown the time-locked suppression of activity in the DMN to a multitude of cognitive, language, and motor events when contrasted with the resting, baseline conditions. ${ }^{3}$

A number of ECoG reports detailing the functional dynamics of the DMN in adult populations have been published. ${ }^{6,12,19,23}$ These studies have observed significantly greater HGP in baseline epochs than in active behavioral epochs. Consequently, task-related HGP suppression has emerged as a key electrophysiological signature of DMN function in adults and is predicted to reflect the task-induced deactivation noted in functional MRI (fMRI) studies. ${ }^{6,12}$

Here, we contrasted task-evoked HGP suppression between children and adults within a lateral node of the DMN to characterize the maturational time frame of evoked HGP suppression across human brain development.

\section{Methods}

Neurophysiological recordings were acquired from 3 young children (age range 3-6 years) and 3 adults (age range 20-38 years) (see Table 1 for demographic information). All participants were undergoing long-term ECoG monitoring for seizure focus localization, and all presented with intractable partial epilepsy with either simple or complex semiology. We included only participants with left-hemisphere frontotemporal and/or temporoparietal ECoG electrode coverage and preoperatively acquired resting-state fMRI (rsfMRI) and ECoG data that were recorded during a structured language task. Electrophysiological and imaging data sets from the children were acquired at Seattle Children's Hospital ( $\mathrm{SCH})$, while data from adult participants were acquired at Harborview Medical Center (HMC) in Seattle, Washington.

For pediatric participants, parents provided informed consent, and assent was obtained from the 6-year-old participant. Informed consent was obtained from adult participants. Consent and assent procedures were conducted in accordance with the guidelines established by the institutional review boards at each institution (SCH and HMC).

All imaging procedures were performed preoperatively using either a Siemens 3-T Trio scanner $(\mathrm{SCH})$ or a Philips 3-T Achieva (HMC) scanner. All scans were acquired using an 8-channel head coil. A standard rsfMRI echoplanar sequence was used to identify the DMN in each participant. The scanning protocol differed slightly depending on the scanner/facility (TE/TR 27/2000 msec, 64

TABLE 1. Summary of participant demographics and cognitive and behavioral data

\begin{tabular}{ccccccc}
\hline Participant No. & Age (yrs) & Sex & Epilepsy Duration (yrs) & Full-Scale IQ & No. of Eliminated Trials* & Voice-Onset Time, msec† \\
\hline S1 & 3.01 & M & 2 & $80 \ddagger, 84 \S$ & NA & NA \\
\hline S2 & 3.5 & M & 2 & $86 \uparrow, 94^{* *}$ & 9 & $1577 \pm 487$ \\
\hline S3 & 6 & F & 4 & $113 \dagger \dagger, 114 \S$ & 6 & $1842 \pm 511$ \\
S4 & 20 & M & 2 & $83 \ddagger \ddagger, 87 \S$ & 0 & $1633 \pm 457$ \\
\hline S5 & 33 & M & 2 & $86 \ddagger \ddagger, 74 \S$ & 0 & $1298 \pm 343$ \\
\hline S6 & 37 & F & 22 & $108 \ddagger \ddagger, 118 \S$ & 5 & $1173 \pm 312$ \\
\hline
\end{tabular}

NA = not applicable.

All participants had left-hemisphere grid coverage.

* Eliminated trials include invalid trials (nonresponse or noncompliant) and/or trials with the presence of interictal spiking.

$\dagger$ Values are shown as the mean \pm SD.

$\ddagger$ Determined using the Wechsler Preschool and Primary Scale of Intelligence IV.

$\S$ Determined using the Verbal Comprehension Index subscale.

II Determined using the Wechsler Preschool and Primary Scale of Intelligence III.

** Determined using the General Language Composite subscale.

†† Determined using the Wechsler Intelligence Scale for Children III.

护 Determined using the Wechsler Adult Intelligence Scale IV. 

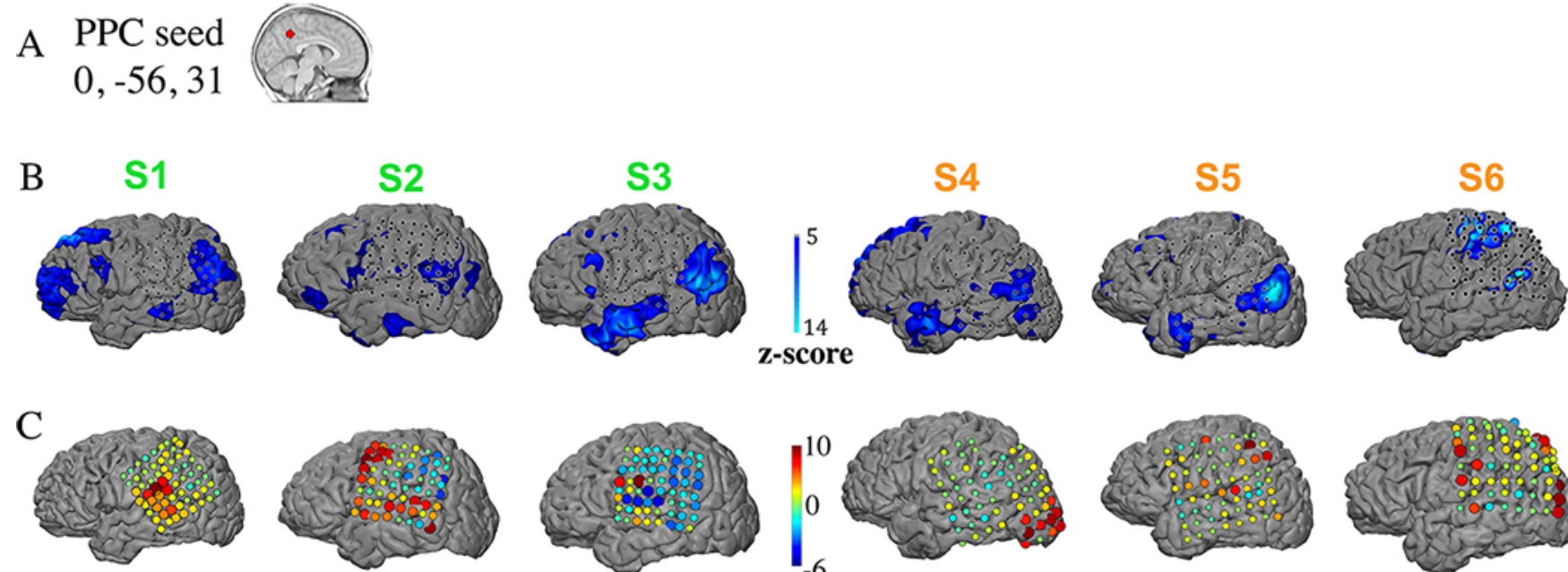

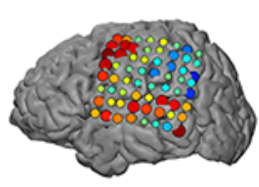

Children

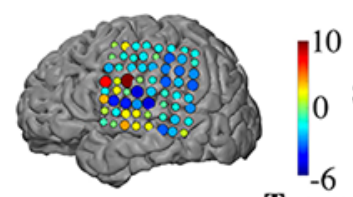

T-score
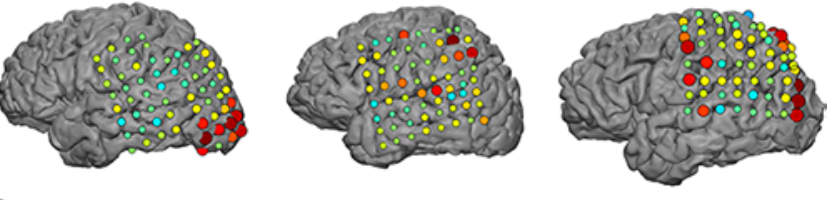

Adults

FIG. 1. DMN electrode identification. A: For each participant, a DMN rsfMRI map was generated using a standard seed point approach. B: The lateral extent of the resulting rsfMRI DMN map (z-scores) for each of the 3 children (S1-S3 in green) and 3 adult (S4-S6 in orange) participants. The coregistered ECoG grids are overlaid on native MRI space. C: Mean HGP (T-scores) during picture naming reveals positive (naming > rest) and negative (naming < rest) responses across electrodes. Electrodes are scaled by both heat map and size to better illuminate the response magnitude. DMN electrodes were selected to 1) overlap with that participant's rsfMRI DMN map and 2) show statistically significant HGP suppression (alpha level < 0.05 ). This process was used solely as a means to identify DMN electrodes rather than formulate statistical interpretations. PPC $=$ precuneus/posterior cingulate. Figure is available in color online only.

$\times 64$ matrix, 192 volumes at SCH; TE/TR 21/2000, $64 \times$ 64 matrix, 200 volumes at HMC). The 2 youngest children (Participants S1 and S2, both 3 years old) were scanned under light propofol anesthesia $(250 \mu \mathrm{g} / \mathrm{kg} / \mathrm{min})$.

Standard rsfMRI preprocessing steps were first implemented in the FMRI Expert Analysis Tool (FEAT; version 5.0), which is part of the FMRIB Software Library (FSL, www.fmrib.ox.ac.uk/fsl), and included motion correction, spatial smoothing using a 6-mm full width at half maximum gaussian kernel, grand mean intensity normalization, and linear drift removal. To limit the effects of noise and motion on blood oxygen level-dependent (BOLD) functional connectivity (FC) estimates, the overall time series was low-pass filtered at $0.2 \mathrm{~Hz}$. We also removed any volume exceeding $0.5 \mathrm{~mm}$ of motion in any direction or plane. This was necessary for Participant S3, who required the elimination of 2 volumes. Each $4 \mathrm{D}$ data set was entered into a multiple regression analysis, and the movement parameters, CSF, white matter, and global signals were treated as nuisance variables.

We used rsfMRI seed point FC to identify and label electrodes placed over the lateral node of the DMN. To construct each participant's DMN FC map, we applied a seed point analysis approach to each rsfMRI data set. An 8-mm spherical seed point was then placed in the precuneus/posterior cingulate (Fig. 1A) of the Montreal Neurological Institute (MNI) standard brain (MNI152) according to standardized published coordinates. ${ }^{2}$ For each participant, an affine registration (FSL MCFLIRT) was used to align the MNI adult template and seed point to an age-appropriate template brain when necessary ${ }^{8}$ and finally to native fMRI space. Seed point DMN rsfMRI maps were generated by voxel-wise regression of the mean time course of all voxels within the precuneus/posterior cingulate seed point using a fixed-effects model. Each map was converted into z-scores using a general linear model approach (FSL FEAT) and corrected for multiple comparisons at the individual level using a cluster correction set at an alpha level of 0.05. rsfMRI DMN maps were then projected onto the brain surface using custom MATLAB scripts that projected the functional data on the T1-weighted space using the isosurface 3D projection algorithm in MATLAB.

All ECoG recordings were acquired from $8 \times 8$ electrode grid arrays with exposed $2.3-\mathrm{mm}$-diameter platinum pads embedded in silastic (AdTech) that were spaced at $7.5 \mathrm{~mm}(\mathrm{~S} 1$ and $\mathrm{S} 2)$ or $1 \mathrm{~cm}$ from center to center (S3S6). The specific placement of the ECoG grids along the lateral temporoparietal surface was determined solely by the clinical indications. Electrode locations were identified and projected onto the T1-weighted MPRAGE cortical surface that was rendered according to our established methods. ${ }^{10}$ Experimental recordings were performed at the participant's bedside with g.USBamps (GugerTec) sampled at $1200 \mathrm{~Hz}$. Cortical potentials were recorded with respect to the epidural reference and ground electrodes (SCH patients) or scalp electrodes (HMC patients). Electrophysiological recordings and the picture-naming task were completed using the general-purpose BCI2000 stimulus and acquisition program. ${ }^{27}$ Hardware imposed filters were set at $0.1 \mathrm{~Hz}$ (high pass) and $500 \mathrm{~Hz}$ (low pass) with a notch filter set at $60 \mathrm{~Hz}$.

The picture-naming task required a vocalized response to the serial presentation of 40 cartoon pictures of common objects (e.g., fork and book). Pictures were presented for a period of $3000 \mathrm{msec}$ with a 3000-msec intertrial 
interval (ITI) rest that was interdigitated between each trial and denoted by the appearance of a centrally located crosshair. Participant S2 performed the beta version of the task where the ITI was $1500 \mathrm{msec}$. All stimuli were shown on an LCD computer monitor at a distance of $75-100 \mathrm{~cm}$ from each participant.

Participant S1 (3 years old) did not verbally respond to stimuli, but he remained relatively alert throughout the task. Thus, the name of each picture was read out loud to him by a researcher. The patient was engaged and relatively attentive to the researcher's interactions. Additionally, parental and/or researcher praise was typically provided throughout the session, and encouragement followed periods of nonresponse for all children. Consequently, we isolated trials containing active, participant-specific, valid responses and eliminated behavioral noise by manually identifying and segmenting (Adobe Audition software, Adobe Systems Inc.) the verbal responses from the picture-naming audio recordings (sampled at $48 \mathrm{kHz}$ ). The edited file was then imported into MATLAB, resampled, and merged with the ECoG recordings to earmark voice onset as an identifiable stimulus trial event. Only valid responses that met the following criteria were included in the statistical analyses: 1) the participant verbally responded, independent of accuracy, within the active trial period prior to the onset of the following ITI, and 2) the stimulus-presenting trial or subsequent ITI was absent of any experimenter or parental encouragement. The number of trials eliminated for each participant varied and is denoted in Table 1.

The ECoG signal processing steps were conducted in the MATLAB computing environment using previously published signal processing approaches to probe adult DMN function. ${ }^{6,9,23}$ Signals were first manually inspected for artifacts and/or significant noise. Channels and/or specific trials showing abnormal interictal discharges were eliminated from all analyses (Table 1). ECoG signals from clean channels were then re-referenced with respect to the common average of the full (grid) montage. Time-series data were notch filtered to eliminate line noise and then band passed into canonical low-frequency components: alpha $(8-12 \mathrm{~Hz})$, beta $(12-30 \mathrm{~Hz})$, and HGP. All filtering steps were performed using fourth-order Butterworth filters. For each band pass, instantaneous power was then computed using the square of the analytical amplitude estimate from the Hilbert transform and log transformed to approximate a normal distribution.

Instantaneous power across frequency bands was normalized to the mean of the full time series from which it came and divided by the standard deviation (z-scores). Importantly, statistical analyses were conducted on these normalized but unsmoothed data sets. For visualization of event-related time series (ERTS), the normalized time series was then smoothed by convolving the data with a 200-msec sliding gaussian window. ${ }^{9}$

DMN electrodes were selected according to 2 predefined criteria: 1) overlap with the DMN's lateral parietal hub as identified on the rsfMRI FC map, and 2) significant reduction in HGP during picture-naming epochs relative to rest. To accomplish this, normalized (z-score) log HGP estimates were binned and averaged (separately for each band) across all valid naming trials and valid resting ITI trials. Statistical maps were generated using the 2-sample Student $\mathrm{t}$-test. Note that this process was used simply to screen electrodes to identity responsive ones, and not draw specific statistical inference.

Next, we statistically contrasted patterns of temporally evolving HGP across the picture-naming task windows using an event-related approach. Previous adult DMN investigations during language tasks have noted peak HGP responses occurring within the 1st second of the rest period. ${ }^{6}$ Thus, we generated the DMN ERTS plots by estimating the percentage of signal change of the mean HGP relative to a 500-msec window of the mean HGP during rest (extracted from the 0.5- to 1.0-second window) (Fig. 2). To test for statistical significance between distributions, we used the 2-sided nonparametric Wilcoxon rank-sum test to correct for multiple comparisons using false-discovery rate correction.

Finally, we examined differences at the group level by binning normalized DMN HGP into six 500-msec windows collapsed across the 2 groups. To determine statistical interactions, we applied 2-way ANOVA using the trial time windows as the within-subject factor and age category as the between-subject factor. The Tukey honest significant difference post hoc test was used to reveal statically significant time window-group interactions. For the qualitative visualization of these group-level effects, we generated cortical activation maps. These maps were generated as previously described. ${ }^{18}$ Briefly, we applied a linear superposition of spherical gaussian kernels $(3 \mathrm{~mm})$ centered at the location of each significant electrode. The kernels were scaled by the HGP activation weight associated with the responsive electrode for a given group and interpolated at each point in the MNI template brain.

\section{Results}

Generally, all participants performed the picture-naming task with little difficulty (see Table 1). However, there was notable behavioral variability between the pediatric and adult participants. As previously discussed, the exception to this was Participant S1, who declined to verbalize responses. Typical for this age, young children exhibited decreased vigilance and were less compliant throughout the recording session. Based on our trial inclusion criteria, the number of nonvalid trials removed for each participant included 9 trials from Participant S2 and 6 trials from Participant S3. The mean voice-onset times of the valid trials (Table 1) were more delayed in children than in adult participants. Finally, 5 trials from Participant S6 were removed from processing due to excessive interictal artifacts.

Based on our selection criteria, at least 1 DMN ECoG electrode was identified for each individual participant (see the blue electrodes in Fig. 1 and electrode localization in Fig. 2). In the case where more than 1 DMN electrode fit these criteria, the responsive electrode with the largest (in absolute magnitude) T-score was used. Figure 2 also reveals the HGP ERTS from each participant's selected DMN electrode for the first $1500 \mathrm{msec}$ of the naming epoch where the 0 point reflects the onset of the picture stim- 


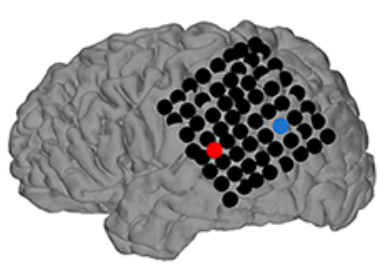

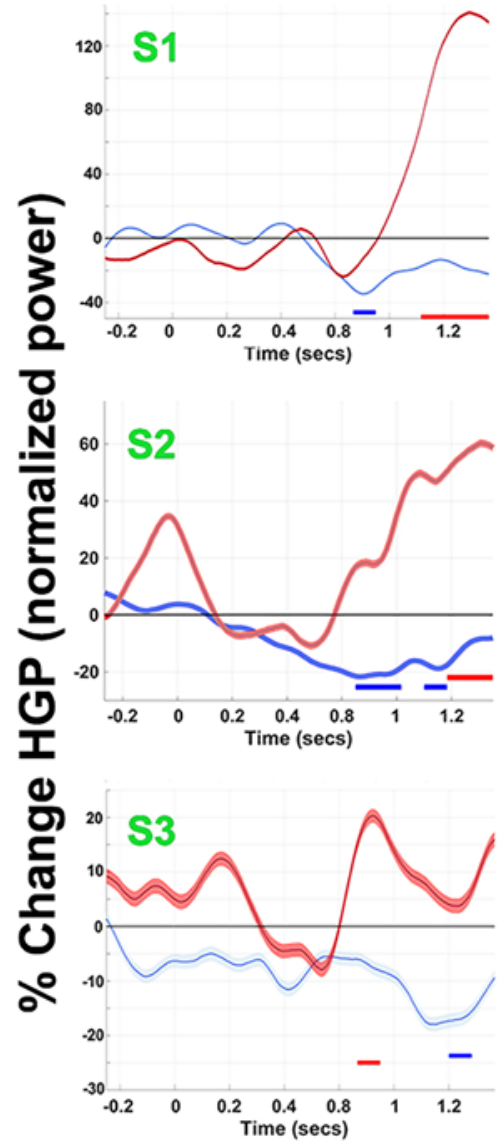

Children
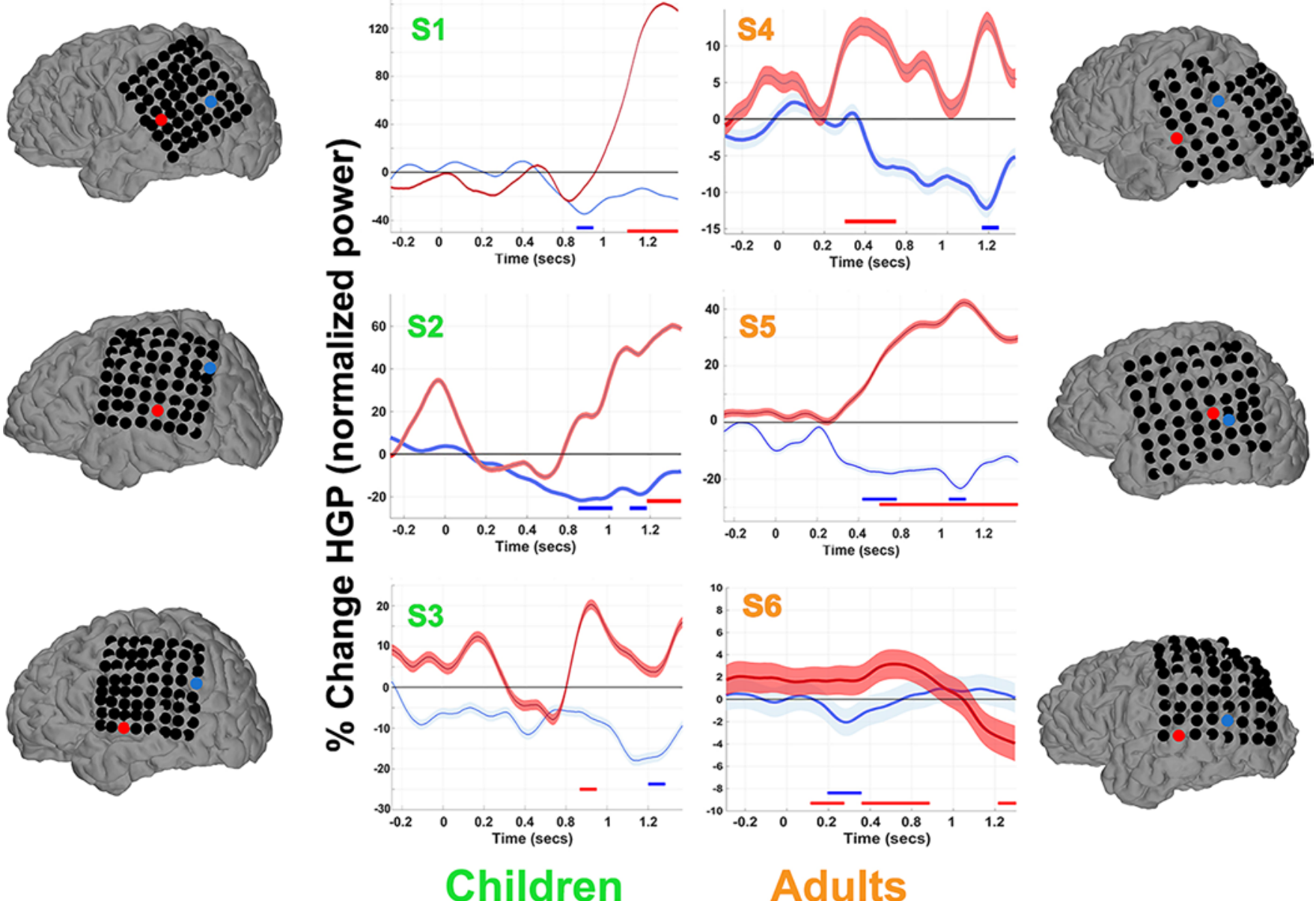

FIG. 2. HGP ERTS plots of selective DMN electrodes (blue) for all 6 participants. Positively responding ERTS electrodes (red) are included simply as a contrast to the DMN's event-related time responses. For each participant, the percentage of the signal change in HGP was calculated based on the second 500-msec time window within the rest period. Dark lines represent the mean ERTS, and shading represents SEM. The 0 point represents the onset of the picture stimulus. Statistically significant periods of HGP during the naming epochs (determined using the 2-sided nonparametric Wilcoxon rank-sum test with false-discovery rate correction for multiple comparisons) are denoted with colored bars below the ERTS plots. Figure is available in color online only.

ulus. We include a positively responding ERTS extracted from an electrode located over the perisylvian language cortex (red electrodes) simply as a frame of reference to the DMN response profile. The blue and red bars denote periods of statistically significant suppression (with respect to rest) and activation, respectively. All participants showed some degree of significant HGP suppression by the identified DMN electrodes. Moreover, the time-evolving peak values of HGP suppression between children and adults according to the DMN ERTS appear to be qualitatively identical. Although we eliminated all behaviorally invalid trials from the analyses, the mean voice-onset times were more delayed in children (Table 1). This is likely related to poorer vigilance and increased environmental distractions in this age group. ${ }^{11}$ Because of these concerns, we did not attempt to statistically contrast peak magnitude estimates of HGP (or any band-limited power estimates) with voiceonset times.

At the group level, our registration process revealed similar electrode-sampling locations on the MNI brain (Fig. 3A). We contrasted the group responses binned into individual time windows spanning the duration of the naming epoch (Fig. 3B and C). This allowed for contrast of HGP modulation at the DMN electrodes aggregated across 105 naming trials for children and 115 trials for adults at 6 different time points. Two-way ANOVA revealed no significant main effect of group $(F[1,1362]=$ $0.169 ; p=0.6634)$ or interaction terms $(F[5,1362]=1.146$; $p=0.3364$ ). Finally, while we observed the consistent suppression of HGP at the DMN electrodes, independent of age group, there was no consistent response pattern for the other frequency bands (Fig. 3D). Specifically, at the DMN electrodes, qualitative beta and alpha band desynchronization was observed in some participants and synchronization in other participants. This is in line with previous evoked DMN power responses spanning an array of canonical frequency bands in adult ECoG participants. ${ }^{23}$

\section{Discussion}

Taken together, these results reveal, for the first time, the task-specific suppression of ECoG HGP in the DMN 


\section{Children Adults}

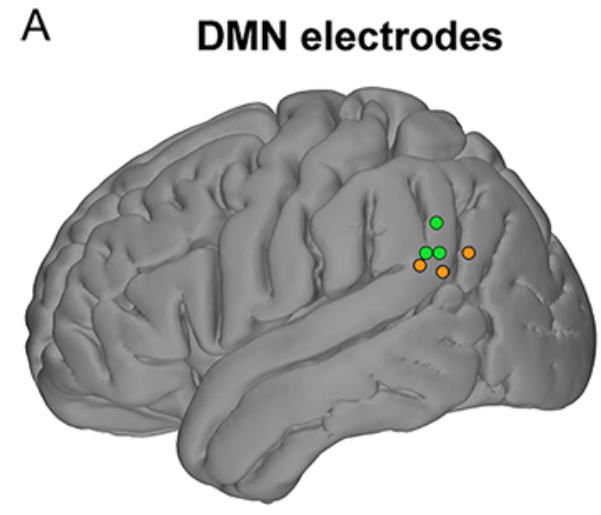

C Naming window time bins

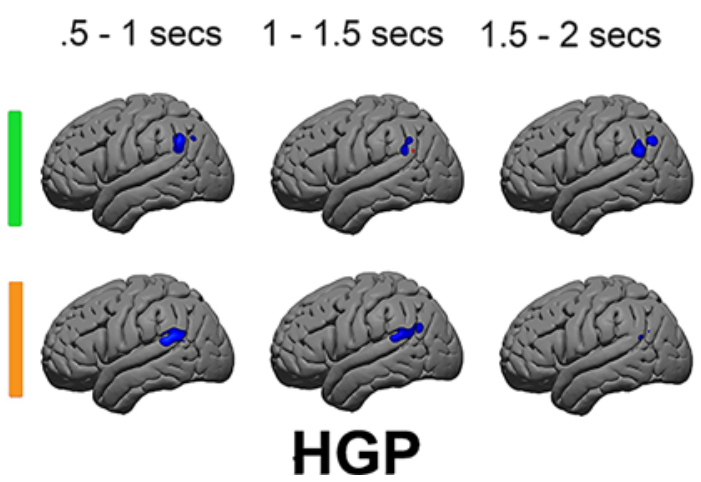

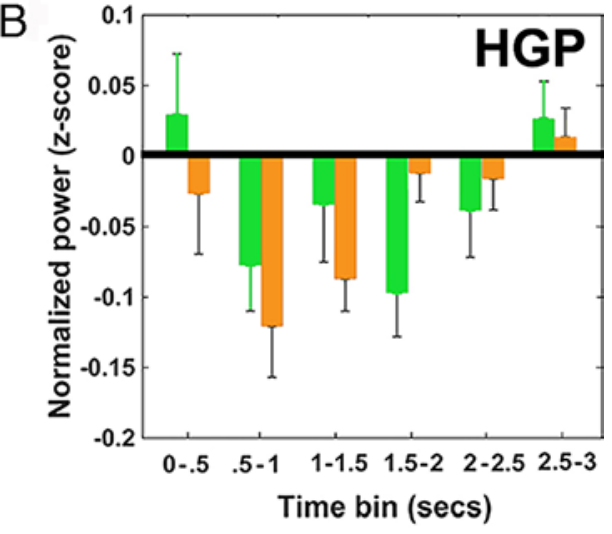

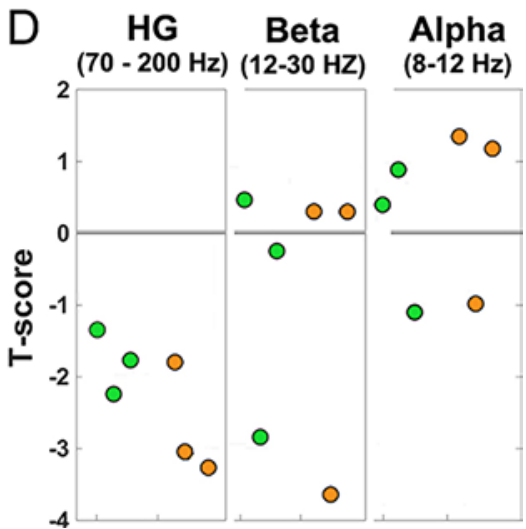

FIG. 3. A: All selected DMN electrodes were registered onto the MNI152 brain, which reveals relatively similar neuroanatomical overlap across participants. B: Mean (across participants) normalized HGP for each of the six 500 -msec time bins spanning the picture-naming epoch. Error bars reflect SEM. There was no statically significant main effect of group or interaction. C: The mean gaussian time plots averaged across the DMN electrodes on both children and adult participants for 3 time bins. D: Only HGP, relative to alpha and beta band power and independent of age, showed a consistent effect of direction under our contrast (naming vs rest) across all participants. Figure is available in color online only.

of young children who are at least 3 years of age (Figs. 2 and 3). This activity showed patterns of evoked HGP suppression that were nearly identical to those that emerged in our adult participants. These observations were extracted from lateral parietal electrodes showing specific overlap with individual rsfMRI-defined DMN components. The direction of evoked HGP within the lateral node of the DMN is consistent with that of the patterns of HG suppression noted in several ECoG reports in adults..$^{6,19,23}$ Based on this signature of DMN function, these results suggest that 1) the lateral parietal hub of the DMN comes online early in human brain development and 2) suppression of HGP is adult-like by at least 3 years of age (if not earlier).

The behavioral noncompliance of Participant S1 should be taken into consideration here. Despite not verbalizing responses, this particular participant remained alert and vigilant during the recording session. He viewed and attended to presented stimuli while listening to the researcher. Using the ECoG data, we noted a lateral parietal electrode that 1) overlapped with the DMN rsfMRI connectivity map and 2) showed consistent, significant HGP suppression during the naming trials. This electro- physiological response was nearly identical to that of the other children and matches other reported patterns of $\mathrm{HG}$ suppression during simple motor tasks. ${ }^{19}$ Taken together, this general pattern is in good agreement with the general notion that DMN suppression is relatively independent of specific goal-directed task requirements. ${ }^{24}$

The combination of ECoG recordings directly from the cortical surface and our spectral decomposition methods provides a robust means for extracting brain-behavior relationships in young children who are notorious for task noncompliance and increased muscle artifacts. The acquisition of the rsfMRI and ECoG data specifically highlights such discrepancies. Both Participants S1 and S2 required sedation prior to imaging procedures, yet we were able to show clear task-evoked electrophysiological modulations using ECoG methodologies without sedation.

Decades of electroencephalography studies have established that oscillatory and synchronized neural activity serves as a primary influence on the maturation and refinement of immature cortical circuitry. ${ }^{29}$ The maturation of various low-frequency activities is linked to the onset of various cognitive and sensorimotor behaviors. For 
example, reports of beta band event-related desynchronization have revealed shifting language dominance from a more bilateral representation in children as young as 5 years to the typical left-hemisphere dominance observed in adults. ${ }^{13}$

Cortical oscillations spanning the neurophysiological spectrum (i.e., high and low spectral processes) are commonly assumed to reflect different functional domains ranging from local cortical computation to mechanisms of long-distance information transfer and the temporal linking of disparate neuronal assemblies. ${ }^{7}$ Furthermore, 1 dominant theory elucidating low-frequency event-related desynchronization is that the suppression of low-frequency oscillations reflects a temporary inhibition of background activity during cognitive and motor processing that facilitates increased excitability of cortical processing neurons. ${ }^{20}$ The general hypothesis related to activity suppression in the DMN is that the temporary shunting of physiological and metabolic resources to a task-positive network function facilitates efficient goal-directed behavior. $^{24}$ Thus, it is conceivable that high-frequency physiology, ${ }^{4}$ including the suppression of HGP noted here, is adequately developed by 3 years of age, normally functions to facilitate cortical computation, and allows for the early acquisition of critical skills. Furthermore, it may be that the flexible cognitive dynamics that define mature behavior do not come online until the complete maturation of the low-frequency mechanisms that link together local activities across the cortical surface. Clearly, future studies need to examine how interactions between various high-frequency measurements pertaining to local cortical parcels and low-frequency response mechanisms serve to facilitate various neurocognitive and behavioral processes across brain development. ${ }^{16}$

\section{Conclusions}

The current results provide an initial step toward understanding the developmental timeline and signature of DMN HGP suppression in the human brain. Because invasive electrophysiological recordings are significantly less influenced by high-frequency cranial muscle artifacts than scalp-based modalities (electroencephalography and magnetoencephalography) and increased noncompliance is characteristic to the pediatric population, these results yield insight into the early timing of this characteristic of cortical high-frequency activity. The findings of this study will not only lay the groundwork for continued investigation into the maturation of high-frequency cortical activity in humans but may also serve to better inform contemporary cognitive and neurobiological models of developmental psychopathology.

\section{Acknowledgments}

The research reported in this paper was supported by grants from the National Institute of Mental Health (R01 NS065186), National Institute of Neurological Disorders and Stroke (MH086118-03 and 1 RC4 NS073008 01), Office of the Director of the National Institutes of Health (NIH K12 2K12HD001097), and National Science Foundation's Engineering Education and Centers (1028725).

\section{References}

1. Anticevic A, Cole MW, Murray JD, Corlett PR, Wang XJ, Krystal JH: The role of default network deactivation in cognition and disease. Trends Cogn Sci 16:584-592, 2012

2. Biswal BB, Mennes M, Zuo XN, Gohel S, Kelly C, Smith SM, et al: Toward discovery science of human brain function. Proc Natl Acad Sci U S A 107:4734-4739, 2010

3. Buckner RL, Andrews-Hanna JR, Schacter DL: The brain's default network: anatomy, function, and relevance to disease. Ann N Y Acad Sci 1124:1-38, 2008

4. Cho-Hisamoto Y, Kojima K, Brown EC, Matsuzaki N, Asano E: Cooing- and babbling-related gamma-oscillations during infancy: intracranial recording. Epilepsy Behav 23:494-496, 2012

5. Crone NE, Korzeniewska A, Franaszczuk PJ: Cortical gamma responses: searching high and low. Int J Psychophysiol 79:9-15, 2011

6. Dastjerdi M, Foster BL, Nasrullah S, Rauschecker AM, Dougherty RF, Townsend JD, et al: Differential electrophysiological response during rest, self-referential, and non-selfreferential tasks in human posteromedial cortex. Proc Natl Acad Sci U S A 108:3023-3028, 2011

7. Donner TH, Siegel M: A framework for local cortical oscillation patterns. Trends Cogn Sci 15:191-199, 2011

8. Fonov V, Evans AC, Botteron K, Almli CR, McKinstry RC, Collins DL: Unbiased average age-appropriate atlases for pediatric studies. Neuroimage 54:313-327, 2011

9. Foster BL, Dastjerdi M, Parvizi J: Neural populations in human posteromedial cortex display opposing responses during memory and numerical processing. Proc Natl Acad Sci U S A 109:15514-15519, 2012

10. Hermes D, Miller KJ, Noordmans HJ, Vansteensel MJ, Ramsey NF: Automated electrocorticographic electrode localization on individually rendered brain surfaces. J Neurosci Methods 185:293-298, 2010

11. Hoehl S, Wahl S: Recording infant ERP data for cognitive research. Dev Neuropsychol 37:187-209, 2012

12. Jerbi K, Vidal JR, Ossandon T, Dalal SS, Jung J, Hoffmann $\mathrm{D}$, et al: Exploring the electrophysiological correlates of the default-mode network with intracerebral EEG. Front Syst Neurosci 4:27, 2010

13. Kadis DS, Pang EW, Mills T, Taylor MJ, McAndrews MP, Smith ML: Characterizing the normal developmental trajectory of expressive language lateralization using magnetoencephalography. J Int Neuropsychol Soc 17:896-904, 2011

14. Kojima K, Brown EC, Rothermel R, Carlson A, Fuerst D, Matsuzaki N, et al: Clinical significance and developmental changes of auditory-language-related gamma activity. Clin Neurophysiol 124:857-869, 2013

15. Lachaux JP, Axmacher N, Mormann F, Halgren E, Crone NE: High-frequency neural activity and human cognition: past, present and possible future of intracranial EEG research. Prog Neurobiol 98:279-301, 2012

16. Maguire MJ, Abel AD: What changes in neural oscillations can reveal about developmental cognitive neuroscience: language development as a case in point. Dev Cogn Neurosci 6:125-136, 2013

17. Miller KJ, Honey CJ, Hermes D, Rao RPN, denNijs M, Ojemann JG: Broadband changes in the cortical surface potential track activation of functionally diverse neuronal populations. Neuroimage 85:711-720, 2014

18. Miller KJ, Leuthardt EC, Schalk G, Rao RPN, Anderson NR, Moran DW, et al: Spectral changes in cortical surface potentials during motor movement. J Neurosci 27:2424-2432, 2007

19. Miller KJ, Weaver KE, Ojemann JG: Direct electrophysiological measurement of human default network areas. Proc Natl Acad Sci U S A 106:12174-12177, 2009

20. Neuper C, Pfurtscheller G: Event-related dynamics of corti- 
cal rhythms: frequency-specific features and functional correlates. Int J Psychophysiol 43:41-58, 2001

21. Nikolić D, Fries P, Singer W: Gamma oscillations: precise temporal coordination without a metronome. Trends Cogn Sci 17:54-55, 2013

22. Olson JD, Wander JD, Johnson L, Sarma D, Weaver K, Novotny EJ, et al: Comparison of subdural and subgaleal recordings of cortical high-gamma activity in humans. Clin Neurophysiol 127:277-284, 2016

23. Ossandón T, Jerbi K, Vidal JR, Bayle DJ, Henaff MA, Jung $\mathrm{J}$, et al: Transient suppression of broadband gamma power in the default-mode network is correlated with task complexity and subject performance. J Neurosci 31:14521-14530, 2011

24. Raichle ME: The brain's default mode network. Annu Rev Neurosci 38:433-447, 2015

25. Raichle ME, MacLeod AM, Snyder AZ, Powers WJ, Gusnard DA, Shulman GL: A default mode of brain function. Proc Natl Acad Sci U S A 98:676-682, 2001

26. Saby JN, Marshall PJ: The utility of EEG band power analysis in the study of infancy and early childhood. Dev Neuropsychol 37:253-273, 2012

27. Schalk G, McFarland DJ, Hinterberger T, Birbaumer N, Wolpaw JR: BCI2000: a general-purpose brain-computer interface (BCI) system. IEEE Trans Biomed Eng 51:10341043, 2004

28. Siegle JH, Pritchett DL, Moore CI: Gamma-range synchronization of fast-spiking interneurons can enhance detection of tactile stimuli. Nat Neurosci 17:1371-1379, 2014
29. Uhlhaas PJ, Roux F, Rodriguez E, Rotarska-Jagiela A, Singer W: Neural synchrony and the development of cortical networks. Trends Cogn Sci 14:72-80, 2010

\section{Disclosures}

The authors report no conflict of interest concerning the materials or methods used in this study or the findings specified in this paper.

\section{Author Contributions}

Conception and design: Weaver, Ojemann. Acquisition of data: Weaver, Poliakov, Novotny, Olson. Analysis and interpretation of data: Weaver, Poliakov, Grabowski. Drafting the article: Weaver, Novotny, Olson. Critically revising the article: all authors. Reviewed submitted version of manuscript: Weaver, Novotny, Olson, Ojemann. Approved the final version of the manuscript on behalf of all authors: Weaver. Statistical analysis: Poliakov. Study supervision: Weaver, Ojemann.

\section{Correspondence}

Kurt E. Weaver, Department of Radiology, University of Washington, 1959 NE Pacific St., Box 357115, Seattle, WA 98195. email: weaverk@uw.edu. 